Gynäkol Geburtschilfliche Rundsch 1995;35:I-IV

\title{
Contents, Vol. 35, 1995
}

\section{Nr.1}

Fortbildung

\section{Editorial 1}

Originalarbeiten

Prävention von Neuralrohrdefekten durch Folsäurezufuhr in

der Frühschwangerschaft

Deutsche Gesellschaften fur Ernährung, Gynäkologie und Geburtshilfe, Humangenetik,

Kinderheilkunde; Gesellschaft fur Neuropädiatrie

Histologische Veränderungen in den unterbundenen Stümpfen 6 der Ovarialgefásse bei präoperativer niedermolekularer Hepa-rinprophylaxe Slunsky, R.

Pathogenese und Diagnostik des polyzystischen Ovarsyndroms 12 Urdl, W.; Desoye, G.

CD44-Splice-Varianten als Prognosefaktoren beim invasiven 16

Zervixkarzinom

Reinthaller, A.; Kainz, C; Kohlberger, P.; Tempfer, C; Gitsch, G;

Kölbl, H.; Breitenecker, G.

Die gynäkologische Untersuchung: Empfindungen, Befürch- 20

tungen und Erwartungen von Poliklinikpatientinnen Vondruska-Benkert, K.; Steiner, R.A.;

Buddeberg, C; Haller, U.

Der klinische Fall

Persistierender Ductus omphaloentericus - Eine mögliche 26

Ursache des blutenden Nabels

Fink, D.; Schär, G; Köchli, O.R.; Perucchini, D.; Haller, U.

Systemische Methotrexattherapie bei Trophoblastpersistenz29

nach tubenerhaltender Operation wegen Extrauteringravidität Perucchini, D.; Schär, G.; Köchli, O.R.; Fink, D.

Management of Severe Blood Loss after Tumor Resection in a 34 Jehovah's Witness Kunz, J.;

Mähr, R.

Transvaginalsonographie eines «Placental Site Trophoblastic 38

Tumor»

Bajka, M.; Köchli, O.R.; Schmidt, D.; Robbiani, M.; Stallmach, T.;

Haller, U.

Spektrum

Gynäkologische Onkologie am Royal Hospital for Women, 42

Sydney. Bericht über einen 1Omonatigen Auslandsaufenthalt Gitsch, G.

Grenzüberschreitungen der Naturheilkunde 46

Lindenmann, J.

Konsensus

Stellungnahme der Deutschen Gesellschaft für Gynäkologie 
und Geburtshilfe zum beabsichtigten Widerruf der Zulassung

CPA-haltiger Präparate

Hammerstein, J.; Rabe, T.; Runnebaum, B.

Stellungnahme der Deutschen Gesellschaft für Endokrinologie

zum Thema Cyproteronazetat

Ständige Kommission für Hormontoxikologie

Synergistischer Effekt von Delta-Aminolävulinsäure und pho- 85 todynamischer Lasertherapie anhand eines In-vitro-Modells mit dem ATP-Tumorchemosensitivitätstest Untch, M.; Korell, M.; Kirschstein, M.; Hepp, H.

Einsatz der photodynamischen Lasertherapie in der 90

Gynäkologie

Korell, M.; Untch, M.; Abels, C; Dellian, M.; Kirschstein, M.;

Baumgartner, R.; Beyer, W.; Goetz, A.E.

Der klinische Fall

Sarkoidose der Mamma. Fallbericht einer Patientin mit 98

isolierter Sarkoidose der Mamma

Döinghaus, K; Dresel, V.; Buhner, M.; Schulz-Wendtland, R.; Lang, N.; Arras, H.; Horn, U. II

Historische Ecke

Zur operativen Behandlung des Mammakarzinoms seit 103

Hippokrates von Kos Wilmanns, J.C.

Konsensus

Empfehlungen für die Anwendung von Prostaglandinen in der 112 Gynäkologie und

Geburtshilfe (Stand: Mai 1994) Dennemark; Dietl, J.; Hick1, E.; Hohmann, M.; Künzel, W.;

Rath, W.; Renziehausen, K.; Schmidt, W.; Schneider, K.T.M.; Weise, W.; Weitzel, H.; Wolff,

F.; Zahradnik, H.P.

Fortbildung

Einleitung 114

Remold, E.

Hysteroskopie - Stellenwert und Risiko. Hysteroskopie versus 114

fraktionierte Kürettage: Die therapeutische Insuffizienz der

Abrasio

Concin, H.; Bösch, H.; Schwärzler, P.

Stellenwert der Hysteroskopie im Rahmen der modernen

Gynäkologie

Deix, R. 116

Doppler-Sonographie des Endometriums unter Hormon-

substitution

Alge, A.; Geringer-Volgger, B.; Solder, E.; Penz-Koza, A.; Marth, C. 118

Fortbildung: Symposium

Einleitung Remold, E. 120

Chirurgischer Standard beim Zervixkarzinom Winter, R. 120

Chirurgischer Standard beim Endometriumkarzinom Marth, C; Dapunt, 0. 122

Chirurgischer Standard beim Ovarialkarzinom Reinthaller, A.; Kölbl, H. 123 
Chirurgischer Standard beim Mammakarzinom Seifert, M.; Vogl, S.; Kubista, E. 126

Veranstaltungskalender 129

Nr.3

Editorial 131

Laudatio auf Prof. Gitsch 132

Originalarbeiten

Zukunftsperspektiven der operativen Gynäkologie bei der Behandlung des Zervixkarzinoms Höckel, M. 133

Die «Wiener Schule» der operativen Gynäkologie als wichtiger 139 Bestandteil moderner

Therapiekonzepte Kölbl, H.

Die vaginal-operative Schule in Österreich. 142

Rückblick - Gegenwart - Zukunft Leodolter, S.

Die Zukunft der operativen Gynäkologie: Vom Dogma zur 149

Wissenschaft - von der Wissenschaft zum Standard Sevelda, P.

Historische Ecke

Das Tor, das der Kaiser den Frauen auftat. 153

Rotenhausgasse (1), Wien - Alservorstadt

Schaller, A.

Alters- und geschlechtsspezifische Unterschiede der Knochen- 159 dichte von 4000 Jahre alten Individuen Frigo, P.; Lang, C; Lauermann, E.; Eppel, W.; Teschler-Nicola, M.; Remold, E.;

Huber, J.C.

Spektrum

Gemeinsames im Rückblick und Ausblick der operativen 164

Gynäkologie zwischen Charité und den Universitätskliniken Wien Gitsch, E.

Konsensus

Aktualisierte FIGO-Stadieneinteilung

169

Bender, H.G.

Fortbildung

Einfluss der Nadelsuspension auf den Kontinenzmechanismus 170 Pieber, D.; Zivkovic, F.;

Tamussino, G.

Urethrovesikale Morphologie bei kontinenten Frauen in $\quad 173$

Spinalanästhesie

Hanzal, E.; Häusler, G.; Sam, C; Helmer, H.; Enzelsberger, H.;

Chiari, A.; Gosch, M.; Kölbl, H.

Fünf-Jahres-Ergebnisse nach Inkontinenzoperation 175

Tamussino, K.; Zivkovic, F.; Pieber, D.; Ralph, G

Kongressberichte

11 th World Congress on Paediatric and Adolescent 177

Gynaecology. 23.-26. Februar 1995, Singapur Tscherne, G.

10. Kongress der European Association of Gynaecologists and 178 Obstetricians (EAGO)

Reinold, E.

Clinical Week at the Gynecology/Obstetrics Department of the 179 Universitätsspital, Zurich

Staff, A.; Mamsen, A.

Gesellschaftsnachrichten

Mitteilungen der Österreichischen Gesellschaft für 180

Gynäkologie und Geburtshilfe (ÖGGG) 
Mitteilungen der Schweizerischen Gesellschaft für 183

Gynäkologie und Geburtshilfe (SGGG)

Brief an die Schriftleitung 158

Buchbesprechung 184

Veranstaltungskalender

185

Nr.4

Der kl'mische Fall

187

Editorial

Originalarbeiten

Koexistenz nervaler Proliferate mit frühen epithelialen 188

Neoplasien und benignen Ektopien der Cervix uteri Bajardi, F.

Der Einfluss endogener Opioide auf die Nebennierenrinden- 194 aktivität bei Frauen mit hypothalamischer Amenorrhö Krause, B.T.; Möller, S.

Erhebung zur Prävalenz von prä- und perimenstruellen Sym- 199 ptomen am weiblichen

Personal eines Universitätsklinikums Gaulrapp, K.; Backe, J.; Steck, T.

Inguinalbestrahlung versus keine Lymphknotentherapie beim 209

kleinen Vulvakarzinom mit klinisch negativen Lymphknoten

(T1, N0-1)

Kucera, H.; Vavra, N.; Kucera, E.

Glukose-, Insulin- und C-Peptid-Kinetik unter Tokolyse mit 215 oralem Fenoterol

Günter, H.-H.; Nitschmann, A.; Balks, H.J.; Wedeking-Schöhl, H.H.; Niesert, S.

Intravesikale Elektrostimulation zur Behandlung von Blasen- 221

funktionsstörangen. Erste Erfahrungen nach gynäkologischen

Operationen

Kölle, D.; Madersbacher, H.; Kiss, G.; Mair, D.

Chronischer Tuboovarialabszess mit Vaginalfistel nach vagi- 226 naler Hysterektomie:

Kombinierte laparoskopisch-vaginale Therapie Rempen, A.

Spektrum

Promotion in den Niederlanden Brezinka, C.

Fortbildung

Toxoplasmosefrischinfektionen in der Steiermark 233

Möse, J.R.; Vander-Möse, A.; Winter, R.; Häusler, M.

Kongressbericht

Kongresse der Niederländischen Gynäkologengesellschaft - 237 erfolgreiche Änderung des

Konzepts Brezinka, C.

Gesellschaftsnachrichten

Mitteilungen der Österreichischen Gesellschaft für 238

Gynäkologie und Geburtshilfe (ÖGGG)

241243244

Veranstaltungskalender Autorenregister Vol. 35, 1995 SachregisterVol. 35, 1995

Suppl. 1 
XIV. Akademische Tagung deutschsprechender Hoch-schullehrer in der Gynäkologie und Geburtshilfe

Graz, 4.-6. Mai 1995

Herausgeber: R. Winter, Graz; E. Reinold, Wien

S. Karger

Verlag für Medizin und Naturwissenschaften

Basel $\cdot$ Freiburg $\cdot$ Paris $\cdot$ London

New York $\cdot$ New Delhi - Bangkok $\cdot$ Singapore

Tokyo $\cdot$ Sydney

Dosierungsangaben von Medikamenten Autoren und Verlag haben alle Anstrengungen

unternom-men, um sicherzustellen, dass Auswahl und Dosierungsangaben von Medikamenten

im vorliegenden Text mit den aktuellen Vorschriften und der Praxis übereinstimmen. Trotzdem

muss der Leser im Hinblick auf den Stand der Forschung, Änderungen staatlicher

Gesetzgebungen und den ununterbrochenen Fluss neuer Forschungsergebnisse bezüglich

Medikamentenwirkung und -nebenwirkungen darauf aufmerksam gemacht werden, dass

unbedingt bei jedem Medikament der Packungsprospekt konsultiert werden muss, um mögliche Änderungen im Hinblick auf Indi-kation und Dosis nicht zu übersehen. Gleiches gilt für spezielle Warnungen und Vorsichtsmassnahmen. Ganz beson-ders gilt dieser Hinweis für empfohlene neue und/oder nur selten gebrauchte Wirkstoffe.

Alle Rechte vorbehalten.

Ohne schriftliche Genehmigung des Verlags dürfen diese Publikation oder Teile daraus nicht in andere Sprachen übersetzt oder in irgendeiner Form mit mechanischen oder elektronischen

Mitteln (einschliesslich Fotokopie, Tonauf-nahme und Mikrokopie) reproduziert oder auf einem Datenträger oder einem Computersystem gespeichert werden.

(C) Copyright 1996 by S. Karger AG, Postfach, CH-4009 Basel (Schweiz) Printed in Switzerland on acid-free paper by Thür AG Offsetdruck, Pratteln

IV 